\title{
Two-tier system of astronomical constants
}

\author{
J.H. Lieske
}

\section{Jet Propulsion Laboratory}

Pasadena, CA 91109 USA

Conventional astronomical standards are applied to a wide range of applications, many of which do not require state-of-the-art precision. Stability of the standards is of great importance, although there is a trend by some in the astronomical community to promote frequent revision of the values of astronomical constants. It is possible to employ stable standards and to differentially correct them by use of the IERS formulation when very precise observations require such action.

The IERS formulation for the transformation from a fixed frame to an ofdate frame involves precession and nutation along with two parameters $\delta \psi$ and $\delta \varepsilon$ which can represent modifications to the standard precession and nutation formulation. The transformation from the mean-of-date frame to the true-ofdate frame in the IERS formulation using rotation matrices is $\mathbf{r}_{\text {true }}=N \mathbf{r}_{\text {Mean }}$ where

$$
N=R_{1}\left(-\varepsilon_{m}-\Delta \varepsilon-\delta \varepsilon\right) R_{3}(-\Delta \psi-\delta \psi) R_{1}\left(\varepsilon_{m}\right)
$$

where $\varepsilon_{m}$ is the mean obliquity, $\Delta \psi$ and $\Delta \varepsilon$ are the nutations in longitude and obliquity, and where $\delta \psi$ and $\delta \varepsilon$ are IERS parameters which allow for changes to the IAU system. They are tabulated in the IERS reports and are generally thought of as observationally-determined values, but the IERS formulation can be used to introduce corrections of an arbitrary nature.

The matrix $N$ also could be written as

$$
N=Q N_{I A U}
$$

where $N_{I A U}$ is the IAU nutation formulation

$$
N_{I A U}=R_{1}\left(-\varepsilon_{m}-\Delta \varepsilon\right) R_{3}(-\Delta \psi) R_{1}\left(\varepsilon_{m}\right)
$$

and where

$$
\begin{aligned}
Q & =R_{1}\left(-\varepsilon_{m}-\Delta \varepsilon-\delta \varepsilon\right) R_{3}(-\delta \psi) R_{1}\left(\varepsilon_{m}+\Delta \varepsilon\right) \\
& \cong\left[\begin{array}{ccc}
1 & -\delta \psi \cos \varepsilon_{t} & -\delta \psi \sin \varepsilon_{t} \\
\delta \psi \cos \varepsilon_{t} & 1 & -\delta \varepsilon \\
\delta \psi \sin \varepsilon_{t} & \delta \varepsilon & 1
\end{array}\right]
\end{aligned}
$$

with

$$
\varepsilon_{t}=\varepsilon_{m}+\Delta \varepsilon
$$

The IERS formulation for $\delta \psi$ and $\delta \varepsilon$ can be employed to introduce differential corrections to the precession and nutation models while retaining a standard model for a longer period of time. In this manner one could employ the standard IAU precession and nutation models and apply corrections when appropriate using the IERS formulation. Such a process would keep global changes to a minimum but would provide a mechanism to use better values for analyzing data when needed. Then after the separation between precession and nutation corrections is clearly understood one could adopt newer IAU models. 